\title{
Erratum to: Low Strength Static Magnetic Field Inhibits the Proliferation, Migration and Adhesion of Human Vascular Smooth Muscle Cells in a Restenosis Model Through Mediating Integrins B1- FAK, $\mathrm{Ca}^{2+}$ Signaling Pathway
}

\author{
Yan Li, ${ }_{1}^{1}$ Li-Qiang Song, ${ }^{2}$ Michael Q. Chen, ${ }^{3}$ Ying-Mei Zhang, ${ }^{1}{ }_{\text {IngXia Li }},{ }^{1}$ Xu-Yang Feng, ${ }^{1}$ \\ Weijie Li, ${ }^{1}$ Wenyi Guo, ${ }^{1}$ Guoliang Jia, ${ }^{1}$ Haichang Wang, ${ }^{1}$ and Jin Yu ${ }^{1}$
}

${ }^{1}$ Department of Cardiovascular Medicine, Xijing Hospital, Fourth Military Medical University, Xi'an 710032, Shaanxi, People's Republic of China; ${ }^{2}$ Department of Respiratory Medicine, Xijing Hospital, Fourth Military Medical University, Xi'an 710032, Shaanxi, People's Republic of China; and ${ }^{3}$ Department of Bioengineering, Stanford University, Stanford, CA 94305-5444, USA

\section{Erratum to: Annals of Biomedical Engineering (2012) 40(12):2611-2618 DOI 10.1007/s10439-012-0602-8}

This erratum is to correct the correspondence author's information which was misspelled on the original publication of this article. It appears correctly here: for this article, address correspondence to Yan $\mathrm{Li}$, Jin $\mathrm{Yu}$ and Haichang Wang, Department of Cardiovascular Medicine, Xijing Hospital, Fourth Military Medical University, Xi'an 710032, Shaanxi, People's Republic of China. Electronic mail: fireman@fmmu.edu. cn,xjyujin@yahoo.com.cn,wanghc@fmmu.edu.cn

Address correspondence to Yan Li, Haichang Wang, and Jin Yu, Department of Cardiovascular Medicine, Xijing Hospital, Fourth Military Medical University, Xi'an 710032, Shaanxi, People's Republic of China. Electronic mail: fireman@fmmu.edu.cn, wanghc@ fmmu.edu.cn, xjyujin@yahoo.com.cn

The online version of the original article can be found under doi: 10.1007/s10439-012-0602-8. 